\title{
Japan explores the boundary between food and medicine
}

Tokyo \& London. Japan's leading cosmetics manufacturer Shiseido is now marketing rice as a health product. This is the first step by Japanese industry to create a new market for foods engineered to have special medical benefits.

Last month, Shiseido became the first company in Japan to win approval from the Ministry of Health and Welfare to sell a "physiologically functional food", defined by new legislation introduced last September. Shiseido's product consists of rice from which the protein globulin has been removed for the benefit of those allergic to it.

For unexplained reasons, allergy to rice has become common in Japan, afflicting thousands of people young and old. The allergy causes unsightly red lesions on the skin covering large areas of the body. The present cure, the avoidance of rice and its products (including sake) in the diet is not welcomed by the Japanese.

Shiseido's engineered rice is one of many products being developed by hundreds of companies expecting to create a new niche in Japan's huge food market. Basic research in the field by university researchers is being supported by a large grant from the Ministry of Education, Science and Culture (MESC).

Shiseido's rice is produced with an enzyme that removes the allergen while retaining 80 per cent of the nutritious rice seed protein. Reconstituted rice is given a glossy surface; its developers claim that its taste is that of ordinary rice and that it prevents allergy in about 70 per cent of patients.

Another newly approved product is low- phosphate milk, produced by Morinaga Milk Company for patients with chronic kidney disorders. Thirteen others are in the final stages of the eight-step approval process (see figure), including oligosaccharide-based foods for regulating intestinal flora, peptidebased foods for regulating mineral absorption and a material based on soya bean protein for regulating blood cholesterol.

According to Soichi Arai of Tokyo University's Department of Agricultural Chemistry, who is a member of one of the ad hoc committees set up by the Ministry of Health and Welfare, "at least 200 companies" are involved in the research and development of physiologically functional foods. Arai says that companies such as Nestlé are also involved. He is surprised that there seems to be less activity in the United States.

Although it is not illegal in Japan to sell products such as these as food, approval allows companies to claim medical benefits on their labels. Critics are worried that the approval process, which takes one to two years, will not be strict enough, but Shiseido's rice was tested on about 2,000 patients before approval.

The interest of the MESC is unusual. Arai heads a team of 57 researchers at 23 universities supported by approximately $¥ 700$ million ( $\$ 6.5$ million) over three years. One of Arai's studies centres on an inhibitor of cysteine proteolysis isolated from rice which can inhibit the proliferation of viruses such as herpes virus on skin and eyes; his group hopes to breed transgenic rice with high levels of the inhibitor.

David Swinbanks \& John O'Brien

\section{Fish-oil miracle additive brings benefits to some}

Tokyo. Japan's interest in health-improvement foods is illustrated by the current boom in sales of a fatty acid component of fishoil, itself the product of people's contentment with their high IQ and their low incidence of heart disease, the curiosity of a fishmonger and the commercial enterprise of a researcher.

Kiyoshi Kondo, head of the Sagami Research Center southwest of Tokyo, explains that suggestions that "the Japanese might be so clever because they eat lots of fish" stimulated research on fish fatty acids and, in particular, on a material called docosahexaenoic acid (DHA), which was shown to reduce blood cholesterol levels and blood pressure and to improve the brain power of rats.

But the finding of a rich source of DHA was a happy accident. A local fish-shop owner asked Kazumagu Yazawa, a researcher at the centre, why his own mashed-up raw tuna was considered to be much more delicious than that of other shops. Analysis showed that the addition of fish eyeball fat from tuna was the explanation, and that these tissues are an unusually rich source of DHA.

Indeed, the orbital fat of tuna and bonito contains $30-40$ per cent of DHA, compared with the 5-10 per cent in other fish and can be easily purified. This has brought down the price of DHA extraction from several tens of thousands of yen for a gram to a few hundred yen (a few dollars).

Yazawa and his institute have linked up with a company to mass produce capsules of the fish oil, which they sell as a food supplement. The 8,000 bottles of capsules sold by the institute at $¥ 18,000$ (US\$160) each represent only a tiny share of the whole DHA market, worth about $¥ 5$ billion ( $\$ 45$ million) a year.

Now the institute is also working with the small company Sano Shokuhin, which has been feeding the oil to chickens to make DHA-rich eggs. Farms all over Japan have followed suit. Yazawa predicts that many other DHA-enriched foods will soon appear at supermarkets and convenience stores. Moreover, clinical trials using DHA are also about to begin at the National Cancer Center in Tokyo because DHA has also shown antitumour activity.

Kondo says that an application for approval of the DHA-enriched foods as "physiologically functional food" is being considered, but booming sales hardly require the stimulus of approval. 